Revista de Metalurgia

Enero-Marzo 2018, 54 (1), e110

ISSN-L: 0034-8570

https://doi.org/10.3989/revmetalm.110

\title{
Estudio de la evolución del espesor en ensayos de Small Punch Test
}

\author{
David Sánchez-Ávila $^{\mathrm{a}, \mathrm{b}, \varpi}$, Rafael Barea ${ }^{\mathrm{b}}$, Nuria Candela ${ }^{\mathrm{c}}$, Marta Álvarez-Leal ${ }^{\mathrm{a}}$, Fernando Carreño $^{\mathrm{a}}$ \\ ${ }^{a}$ Dpto. de Metalurgia Física, Centro Nacional de Investigaciones Metalúrgicas (CENIM, CSIC). Avda. Gregorio del Amo 8, \\ 28040 Madrid, España \\ ${ }^{\mathrm{b}}$ Dpto. de Ingeniería Industrial, Universidad Nebrija, Escuela Politécnica Superior y de Ingeniería, C/Pirineos 55, 28040 \\ Madrid, España \\ ${ }^{c}$ ESNE, Escuela Universitaria de Diseño, Innovación y Tecnología, Avda. Alfonso XIII 97, 28016 Madrid, España \\ ${ }^{\square}$ Autor para la correspondencia: d.sanchezav@gmail.com
}

Enviado: 23 Mayo 2017; Aceptado: 30 Noviembre 2017; Publicado on-Line: 19 Marzo 2018

\begin{abstract}
RESUMEN: El ensayo de punzonado de probetas miniaturizadas (Small Punch Test, SPT) es un ensayo en creciente expansión empleado para la obtención de diversos datos mecánicos, por ejemplo, de resistencia, fractura, fluencia, etc... especialmente cuando se dispone de poco material. Sin embargo, el ensayo SPT es más complicado que el ensayo de tracción uniaxial dada su no linealidad dificultando la relación de los datos obtenidos con los de tracción. De hecho, en la literatura no hay un modelo evidente que relacione estos ensayos y se debe recurrir a una calibración para cada material. Una de las causas de la complicación del ensayo SPT es que la reducción del espesor de la probeta, según procede el ensayo, no es homogénea en su zona de deformación, a diferencia de tracción. En este trabajo se ha procedido a determinar la variación del espesor de la probeta de SPT en varios puntos, y especialmente en el centro y la zona de rotura, mediante el empleo de elementos finitos en COMSOL tomando como material base un acero 316L obtenido por fabricación aditiva mediante "Selective Laser Melting" (SLM). Para el adecuado modelado en COMSOL se han empleado además los parámetros mecánicos de dos tratamientos termomecánicos extremos del 316L, uno recocido a mínima dureza y otro endurecido por deformación hasta elevada resistencia. Los resultados obtenidos de variación del espesor de la probeta permiten avanzar en el modelado teórico del comportamiento del ensayo SPT para la obtención más precisa de los datos mecánicos equivalentes a ensayos de tracción.
\end{abstract}

PALABRAS CLAVE: Acero 316L; Ensayo de punzonado de probetas miniaturizadas (SPT); Fabricación aditiva con haz láser (SLM); Modelo por elementos finitos (MEF); Variación de espesor de probeta durante ensayo

Citar como/Citation: Sánchez-Ávila, D.; Barea, R.; Candela, N.; Álvarez-Leal; M., Carreño, F. (2018) "Estudio de la evolución del espesor en ensayos de Small Punch Test". Rev. Metal. 54(1): e110. https://doi.org/10.3989/revmetalm.110

\begin{abstract}
Study of the thickness evolution during SPT Testing. The Small Punch Test (SPT) is an increasingly expanding test used to obtain different mechanical data, such as strength, fracture, creep, etc...especially when there is little material available. However, the SPT test is more complicated than the uniaxial tensile test due to its non-linearity, which makes it difficult to relate the data obtained with the tensile tests. In fact, in the literature there is no clear model linking these tests and a different calibration should be used for each material. The complication of the SPT test is that the reduction of the sample thickness is not homogeneous in its gauge volume. In this work we proceeded to determine the variation of the SPT specimen thickness at several points, especially at the center and at the rupture zone, by means of the use of finite elements in COMSOL, taking a SLM AM (selective laser melting additive manufactured) 316L stainless steel as the base material for modelling. For the appropriate modelling in COMSOL, the mechanical parameters of two $316 \mathrm{~L}$ extreme thermomechanical treatments have been implemented, one annealed to a minimum hardness and another heavily work-hardened. The sample thickness variation results allow advancing in the theoretical modeling of the SPT behavior in order to obtain more accurate correlations with tensile tests data.
\end{abstract}

KEYWORDS: 316L stainless steel; FEM (Finite Elements Modelling); SLM (Selective Laser Melting) additive manufacturing; SPT (Small Punch Test); Thickness variation

ORCID: David Sánchez-Ávila (https://orcid.org/0000-0001-6454-6510); Rafael Barea (https://orcid.org/0000-00026784-6110); Nuria Candela (https://orcid.org/0000-0002-0568-753X); Marta Álvarez-Leal (https://orcid.org/00000002-9531-1404); Fernando Carreño (https://orcid.org/0000-0003-0754-2518)

Copyright: (C) 2018 CSIC. Este es un artículo de acceso abierto distribuido bajo los términos de la licencia de uso y distribución Creative Commons Reconocimiento 4.0 Internacional (CC BY 4.0). 


\section{INTRODUCCIÓN}

El 316L es un acero inoxidable austenítico muy extendido en la industria debido a su buen comportamiento en servicio a altas temperaturas o en contacto con medios altamente corrosivos. Además, presenta un gran abanico de posibilidades en cuanto a propiedades mecánicas. Por ello, su estudio sería útil también para analizar el comportamiento mecánico de otros aceros también demandados (Medina et al., 2015; Alcelay et al., 2016; Cisneros-Belmonte et al., 2016; Sevim, 2016; Zhao et al., 2016; Gu et al., 2017; Strbac et al., 2017). La gran cantidad de estudios publicados en torno a esta aleación y su uso continuado hacen que sea el candidato idóneo para un estudio del comportamiento del material durante un ensayo de Small Punch Test, SPT (Puchi-Cabrera et al., 2007; Pascual et al., 2010; Tsisar et al, 2014; Zhong et al., 2017).

La próxima generación de reactores nucleares, fuentes de neutrones y dispositivos de prueba de fusión plantea serios problemas para los componentes estructurales cerca de las fuentes de irradiación por lo que se crea la necesidad del desarrollo de nuevos materiales y ensayos. La caracterización común para la determinación de los parámetros que rigen el comportamiento mecánico se realiza generalmente mediante ensayos de tracción, ya que es el ensayo más sencillo y exigente para un material, en el cual se aplica una tensión uniaxial sobre una longitud de trabajo homogénea definida por la probeta mecanizada a tal efecto. No obstante, muchas veces existen ciertas limitaciones que llevan al uso de otras técnicas. Una importante limitación en la caracterización de materiales de coladas experimentales es el volumen de material disponible para los ensayos. Por otro lado, existe un riesgo con el manejo de muestras irradiadas, con lo cual minimizando el tamaño se minimiza la exposición del personal. Es por esto que se ha llegado al desarrollo de nuevos ensayos para muestras miniatura. Uno de estos ensayos es conocido como ensayo miniatura de punzonado o Small Punch Test (SPT) desarrollado a principios de 1980 por Manahan et al. (1981). Su finalidad es la de estimar el límite elástico, la tensión máxima, la transición dúctil-frágil, propiedades de fluencia, análisis de la anisotropía y tenacidad a la fractura (Mao y Takahashi, 1987; Fleury y Ha, 1998; Díaz et al., 2009; García-Infanta et al., 2009; García et al., 2014; Saucedo-Muñoz et al., 2015).

El ensayo SPT consiste en la flexión de un disco encastrado por la zona exterior y empujado por su centro mediante una bola esférica cuya proyección circunferencial es concéntrica al disco deflectado como se muestra en la Fig.1 (García-Infanta et al., 2009). La aplicabilidad de este ensayo es muy diverso (materiales metálicos, poliméricos y cerámicos), aunque también posee algunas desventajas. Una de ellas es la ausencia de normativa aunque existe un código europeo con algunas recomendaciones

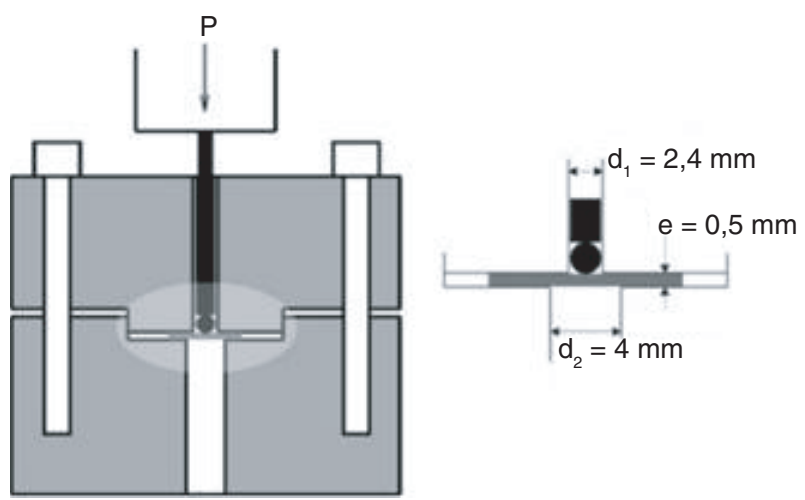

Figura 1. Esquema del utillaje de Small Punch Test y dimensiones del sistema de punzonado y la muestra.

de cómo ha de llevarse a cabo (CEN Workshop Agreement, 2006).

Si bien ya se han realizado y se están llevando a cabo muchos ensayos SPT en materiales de distintas naturalezas como polímeros, cerámicos y metálicos (Rasche et al., 2014; Rodríguez et al., 2016; Arroyo et al., 2017) y bajo diferentes condiciones de velocidad y temperatura, la correlación con los datos provenientes de ensayos de tracción deja mucho que desear. Así, no hay un acuerdo claro con la obtención del valor del límite elástico, la resistencia máxima o la temperatura de transición dúctilfrágil obteniéndose grandes dispersiones (Misawa et al., 1987; Autillo et al., 2006; García et al., 2014; Altstadt et al., 2016a; Altstadt et al., 2016b; Yang et al., 2017). Asimismo, es complicado predecir los valores de ductilidad mediante el ensayo SPT, y por tanto, deben obtenerse directamente de la medición del espesor de la probeta después de ensayar.

En este artículo se estudia la variación del espesor de la probeta en el ensayo de SPT, mediante elementos finitos, en aceros $316 \mathrm{~L}$ con comportamientos muy dispares, abarcando desde recocido de mínima dureza hasta severamente deformado de alta resistencia. El resultado se ha validado experimentalmente con un acero 316L obtenido por SLM con propiedades intermedias.

\section{MATERIALES Y MÉTODOS}

\subsection{Material}

El material ensayado para la validación del modelo de elementos finitos (MEF) es un acero inoxidable austenítico $316 \mathrm{~L}$ procesado mediante fabricación aditiva por fusión láser (SLM) en una atmosfera de Argón. Se fabricaron directamente probetas de tracción planas (tipo hueso de perro) con dimensiones de trabajo $3 \times 3 \times 10 \mathrm{~mm}^{3}$ y radio $3 \mathrm{~mm}$ de ajuste a mordazas, que fueron pulidas por ambas caras para eliminar su rugosidad. De esta fabricación, a fin de validar el modelo de elementos finitos, se ha obtenido la curva 
de tracción mostrada en la Fig. 2. Además, se muestran en esta figura los dos comportamientos teóricos extremos en cuanto a ensayo de tracción, relativos a acero $316 \mathrm{~L}$ de baja resistencia y elevado alargamiento frente a $316 \mathrm{~L}$ severamente deformado de alta resistencia y escaso alargamiento (316L Recocido y 316L Endurecido, respectivamente) utilizados en la alimentación del modelo de elementos finitos. Como se puede ver en la Fig. 2, el acero 316L SLM presenta un comportamiento mecánico intermedio en comparación con los dos extremos de $316 \mathrm{~L}$ estudiados. Estos comportamientos extremos definirán los límites del comportamiento en cuanto al ensayo SPT y permitirán predecir las variaciones de espesores de aceros $316 \mathrm{~L}$ en condiciones intermedias acotadas. Merece la pena destacar que el acero 316L es uno de los materiales metálicos con mayor rango de variabilidad de estados intermedios entre mínima y máxima resistencia por su gran capacidad de endurecimiento por deformación. Esto hace que sea un material muy apropiado para realizar simulaciones y ensayos ya que su variabilidad pone a prueba la consistencia de los resultados obtenidos.

\subsection{Método experimental (Ensayo de Small Punch Test)}

En la Fig. 1 se muestra un esquema del utillaje utilizado para la realización del ensayo SPT (GarcíaInfanta et al., 2009). El módulo de SPT es adaptado lo más rígidamente posible a una máquina universal de ensayos mecánicos Servosis equipada con una célula de carga de $10 \mathrm{kN}$. La geometría de las probetas viene definida en el "Code of Practice" (CEN Workshop Agreement, 2006) 0,500 $\pm 0,05 \mathrm{~mm}$ de espesor donde la bola cuenta con un diámetro de $2,4 \mathrm{~mm}$ y el hueco donde la muestra apoya tiene $2 \mathrm{~mm}$ de radio. Las probetas de $6 \mathrm{~mm}$ de diámetro, son desbastadas de acuerdo al CEN (200-2500 Grit). Estas probetas se obtuvieron de las cabezas de las probetas de tracción. Los ensayos han sido realizados a una velocidad de

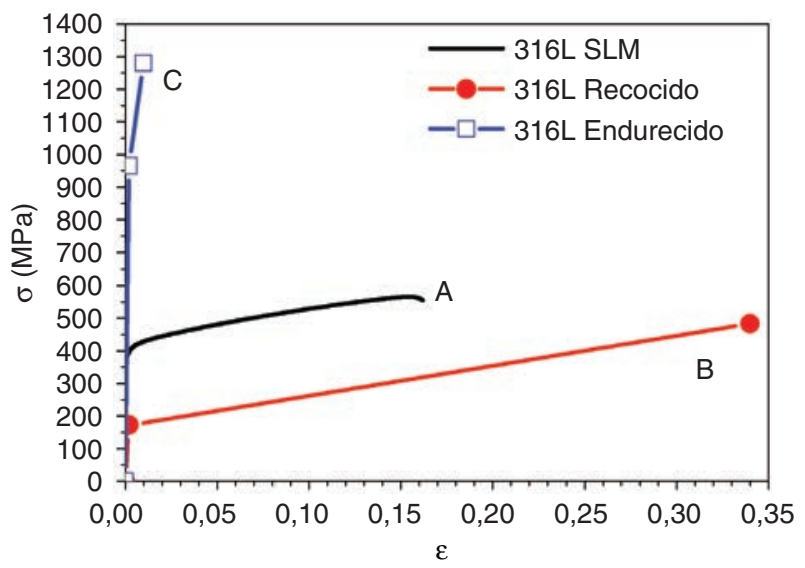

Figura 2. Curvas de tracción 316L: a) 316L SLM, b) 316L Recocido y c) 316L Endurecido. desplazamiento del punzón constante de $0,04 \mathrm{~mm} \cdot \mathrm{s}^{-1}$ y temperatura ambiente. El desplazamiento medido es el del travesaño que desplaza el punzón, con una resolución de $1 \mu \mathrm{m}$. De este ensayo se obtiene la curva fuerza-desplazamiento del 316L SLM y la probeta deformada, la cual es seccionada posteriormente para compararla con el modelo de MEF.

\subsection{Ensayo de Small Punch Test mediante elementos finitos}

El modelado del ensayo se realiza en el software de elementos finitos COMSOL para la determinación de la variación del espesor durante el ensayo de SPT. Es un modelo 2D-axisimétrico estacionario que resuelve el problema para cada desplazamiento vertical, donde se implementan también de forma paramétrica diferentes curvas teóricas de endurecimiento que hacen referencia a dos aceros $316 \mathrm{~L}$ comercializados, uno de ellos severamente endurecido $\left(\sigma_{\mathrm{Y}}=965 \mathrm{MPa}, \sigma_{\mathrm{UTS}}=1280 \mathrm{MPa}, \mathrm{e}=1 \%\right)$ y otro recocido $\left(\sigma_{\mathrm{Y}}=172 \mathrm{MPa}, \sigma_{\mathrm{UTS}}=480 \mathrm{MPa}, \mathrm{e}=40 \%\right)$ mostradas en la Figs. $2 \mathrm{~b}$ y $2 \mathrm{c}$ respectivamente. Estas curvas de tracción se introducen en la simulación siguiendo un modelo elastoplástico sometido a grandes deformaciones y con la condición adicional de prolongar el valor del UTS hasta deformaciones $\varepsilon=2$ para evitar fractura prematura y garantizar la convergencia de la simulación. En general, el acero 316L mostrará comportamientos mecánicos intermedios dependiendo de su historia termomecánica. Así, mediante esta simulación se obtendrán los valores límite de la variación de espesor entre los que se encontrará un acero $316 \mathrm{~L}$ concreto. Con la finalidad de validar el modelo se han implementado ambas curvas de tracción o de endurecimiento, asumiendo en este modelo la bola como infinitamente rígida y considerando contactos al igual que otros autores (Zhou et al., 2013; Haroush et al., 2015; Simonovski et al., 2017) utilizando un coeficiente de fricción de 0,4 . Se han realizado ensayos con diferentes mallados hasta obtener unos resultados independientes del tamaño de la malla. Los cálculos se han realizado bajo el supuesto de grandes deformaciones donde el mallado realizado sobre una sección del disco es de 900 elementos rectangulares que cubren un espesor de $500 \mu \mathrm{m}$ y $3 \mathrm{~mm}$ de longitud, distribuidos con una densidad del mallado gobernada por la física, de forma que hay una mayor densidad de elementos en la zona de mayor gradiente de tensiones.

\section{RESULTADOS Y DISCUSIÓN}

\subsection{Ensayos experimentales a velocidad constante de indentación}

Se han llevado a cabo ensayos de SPT en probetas de acero 316L fabricadas por SLM como se ha descrito en el apartado de experimental. Estas probetas 
sometidas a deformación serán fundamentales para comparar el perfil de variación del espesor de las probetas ensayadas con el obtenido de las simulaciones. En la Fig. 3 se muestra la curva carga - desplazamiento de la bola resultante del ensayo SPT del acero 316L SLM, así como las curvas SPT de los aceros recocido y endurecido obtenidos de la modelización por elementos finitos. Como se observa en la figura, los aceros recocido y endurecido presentan una gran diferencia en su comportamiento de carga soportada por SPT, y el comportamiento del acero 316L SLM se sitúa entre ambos, como cabía esperar. Ahora bien, este artículo no se centra en el comportamiento a carga, sino en la variación de espesor del material ensayado mediante SPT, asunto al que la literatura no le ha prestado especial atención. Para estudiar la variación de espesor se realizó el corte transversal de las probetas ensayadas, comportándose éstas como un caso intermedio en cuanto a propiedades mecánicas entre los dos extremos modelados del 316L (Fig. 2). La Fig. 4 muestra el perfil de una probeta SPT ensayada hasta rotura obtenido mediante microscopía óptica. Se observa en ambos lados de la probeta ensayada la simetría propia del ensayo. De esta figura se obtienen mediciones experimentales

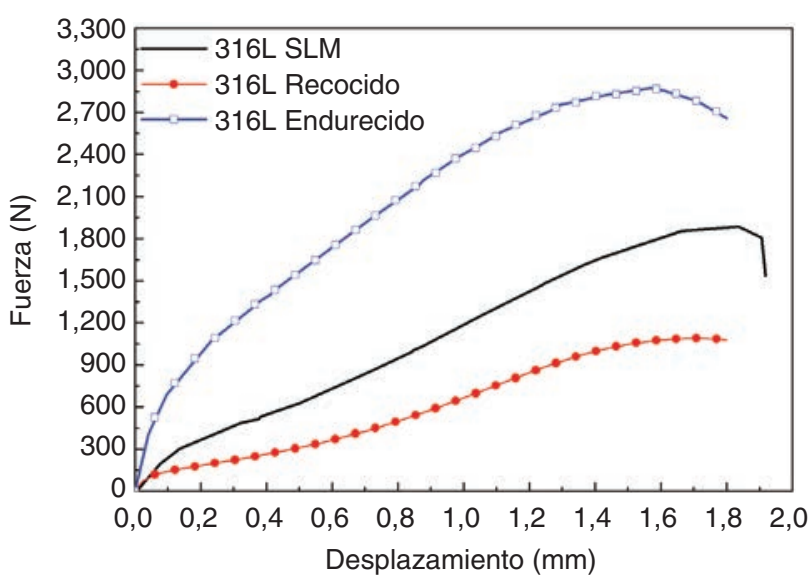

Figura 3. Curvas de SPT del acero 316L SLM, 316L Endurecido y $316 \mathrm{~L}$ Recocido. de los valores de espesor de la probeta. Los valores relativos a la zona central, o copete, han servido para validar el modelo de elementos finitos. Estos valores de espesor experimental en el centro de la probeta una vez ensayada se sitúan en $346 \pm 12 \mu \mathrm{m}$.

Son muchos los estudios en los que se ha analizado la curva carga - desplazamiento distinguiendo varias zonas (Mao y Takahashi, 1987; Rodríguez y Belzunce, 2012; Martínez-Pañeda et al., 2016), pero no se le ha prestado tanta atención a la morfología de la probeta según se ensaya en SPT. En opinión de los autores este aspecto es de gran importancia, de hecho clave, para la correcta interpretación y posterior correlación de los datos de SPT con los de tracción. Puesto que la deformación de la probeta SPT no es homogénea, es necesario conocer en qué zona se produce el grueso de la deformación y el espesor que sustenta la carga de la probeta en cada momento. La Fig. 4 muestra varias características importantes del ensayo de SPT. La primera es que el ensayo produce deformaciones heterogéneas a lo largo de la probeta. Este hecho contrasta con un típico ensayo de tracción en el que la deformación plástica se distribuye homogéneamente a lo largo de toda la longitud (y volumen) de trabajo, desde el límite elástico (YS) a resistencia máxima (UTS), a partir de la cual se produce la estricción. Por ello, el volumen de trabajo "eficaz" de la probeta SPT no está claramente definido, y es claramente menor que el volumen descrito por el anillo que define el agujero del soporte. Este hecho dificulta la adecuada conversión de los datos mecánicos de un ensayo SPT a uno de tracción, y pone de relieve que modelos sencillos teóricos propuestos asumiendo geometrías sencillas son muy aproximados y no suficientemente precisos (Mao y Takahashi, 1987; García-Infanta, et al., 2009; Rodríguez y Belzunce, 2012; Martínez-Pañeda et al., 2016; Yang et al., 2017). Una segunda característica es que los modos de ensayo son distintos en el copete, apoyos y zona de rotura. Al comienzo del ensayo las componentes y modos de deformación están mezclados, dándose una pequeña parte de penetración (tipo dureza), más una gran componente de flexión, que va progresando hacia tracción en la zona de rotura.

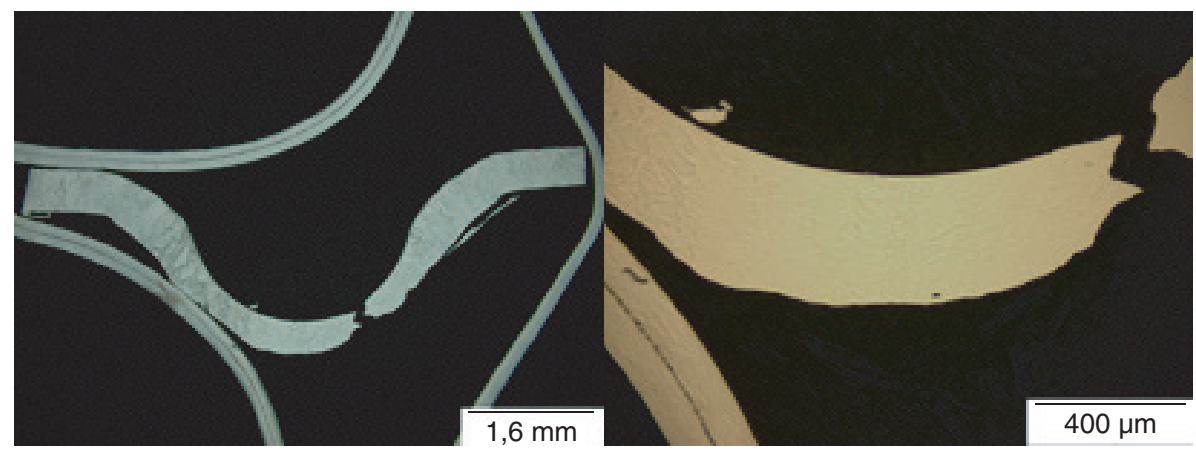

Figura 4. Sección de una Probeta de Small Punch Test 316L SLM ensayada. 


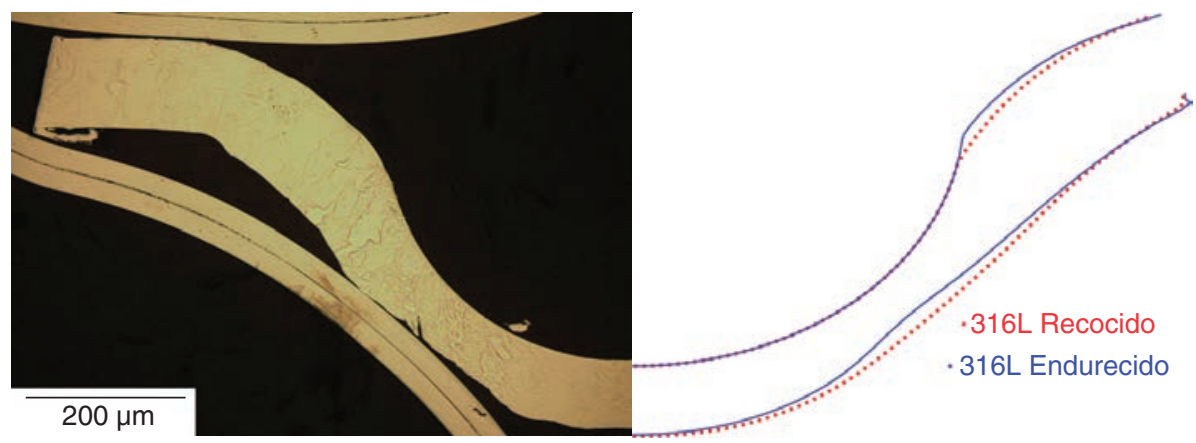

FIGURA 5. Comparación de perfiles de espesor entre MEF y ensayo experimental de 316L SLM.

Por eso en el ensayo SPT no está definido con claridad un YS y se suelen tomar diferentes criterios para ajustar o calibrar a un valor razonable dado por el ensayo de tracción (Lucas et al., 1986; Autillo et al., 2006; CEN Workshop Agreement, 2006; Du et al., 2010). En tercer lugar, no todas las zonas contribuyen de la misma manera a la deformación de la probeta, especialmente la zona cercana a los apoyos (operando más en flexión que tracción) que permanece con el grosor inicial, no aportando material, hasta casi el contacto con la bola. Estas razones evidencian la complejidad del ensayo SPT y su dificultad para la conversión de los datos mecánicos obtenidos en datos equivalentes de tracción.

La simulación MEF realizada introduciendo las curvas de tracción del acero 316L permite obtener la variación morfológica de la probeta en función del desplazamiento de la bola. En la Fig. 5 se comparan el perfil experimental con los dos simulados. Se observa un gran acuerdo a lo largo de toda la probeta desde el copete hasta los apoyos ("hombro"), pasando por la zona de mayor estricción. De hecho, la diferencia de comportamiento entre el acero recocido y el endurecido no es aparentemente muy grande, pero en línea con lo esperado ya que un mayor endurecimiento por deformación (el caso recocido) permite una mayor alimentación del material colindante a medida que deforma, homogeneizando más la deformación que en el caso endurecido, que estricciona y fractura a menor desplazamiento.

En la Fig. 6 se muestra el espesor en la parte central del disco obtenido mediante MEF en función del desplazamiento de la bola tanto para el caso recocido como para el endurecido. Inicialmente se observa una disminución atribuida a penetración de la bola, seguida de dos zonas con pendientes distintas para terminar girando asintóticamente paralelo al eje de abscisas. Tiene sentido este giro asintótico ya que el copete tendería a espesor nulo o muy bajo para penetraciones muy elevadas. Ahora bien, penetraciones mayores a $1,4 \mathrm{~mm}$ se pueden considerar como elevadas, propios de materiales dúctiles y que rompen como el presente acero 316L SLM, mediante estricción que se propaga anularmente.

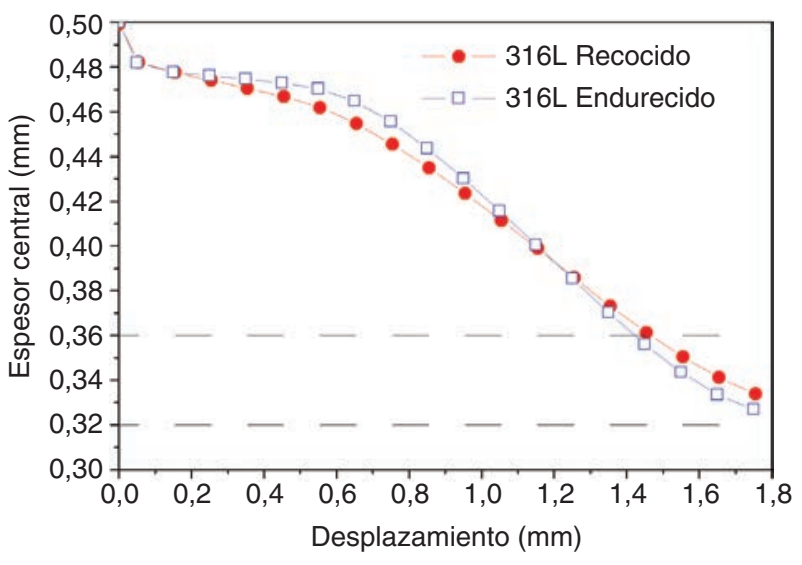

FIgURA 6. Variación del espesor en la parte central de la probeta según el modelo de elementos finitos.

Los materiales menos dúctiles, que muestran fractura prematura debida a defectos importantes, o de muy poca plasticidad, rompen incluso antes de $1,2 \mathrm{~mm}$ de desplazamiento y muestran, en general, una fractura consistente en varias grietas perpendiculares a la fractura dúctil anular (Ma y Yoon, 2010; Turba et al., 2013; Altstadt et al., 2016a). La Fig. 6 muestra que para los valores de desplazamiento de la bola similares a los obtenidos por la probeta experimental los valores de espesor de las simulaciones para el 316L Recocido y 316L Endurecido caen dentro de las mediciones experimentales, aproximadamente a $346 \pm 12 \mu \mathrm{m}$. Esta concordancia valida el modelo de elementos finitos tanto por el hecho de estimar correctamente una mayor variación final del material endurecido respecto al recocido, como por el hecho de predecir el espesor final de la zona central, como han sugerido otros autores (Haroush et al., 2015; Moreno et al., 2016).

\subsection{Determinación de la variación del espesor en la zona de rotura}

La Fig. 7 muestra dos situaciones de la evolución de la morfología de la probeta deformada a $0,5 \mathrm{y}$ $1,5 \mathrm{~mm}$ de desplazamiento de la bola para el 316L 


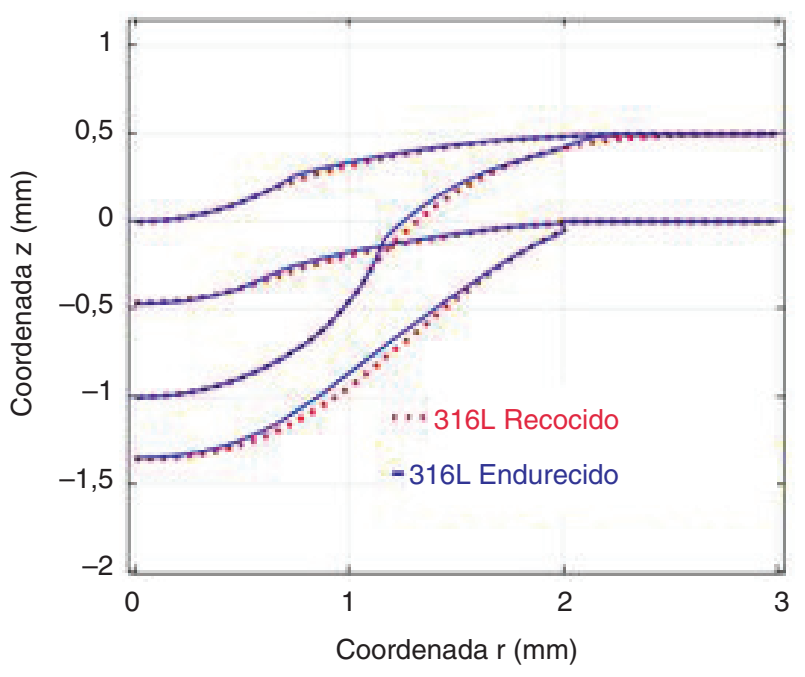

Figura 7. Evolución del perfil de espesor de la probeta para 0,5 y $1,5 \mathrm{~mm}$ de desplazamiento para $316 \mathrm{~L}$ Recocido y $316 \mathrm{~L}$ Endurecido.

Recocido (línea roja) y 316L Endurecido (línea azul). Aunque existe gran diferencia de comportamiento en tracción entre ambos, el espesor predicho por la simulación de las probetas SPT no es, aparentemente, muy distinto. Ahora bien, existen diferencias que son mayores en la zona de rotura, menores en la zona central (copete), y menores aún en la zona de los apoyos (hombro). La zona de mayor interés es la zona de rotura, situada aproximadamente a mitad de camino entre el copete y el hombro, y que muestra una dependencia con la capacidad de endurecimiento de la aleación. Esto es de esperar ya que una diferencia grande entre límite elástico (YS) y resistencia máxima (UTS) provoca una mayor homogeneización de la deformación plástica del material y mayor resistencia a la formación de cuellos (necking). En la Fig. 5 ya se mostró una comparación de los dos comportamientos predichos, endurecido y recocido, con el caso de probeta real para similar desplazamiento. Fijándonos más en la zona de rotura se observa un cuello en la probeta endurecida (línea continua azul). Este es un detalle importante pues, por un lado, muestra que el modelo es capaz de predecir razonablemente hasta donde deformará la probeta, y por otro, permitirá obtener un criterio de cuando emergerá un cuello.

De los diferentes perfiles de las probetas en función del desplazamiento se determina el mínimo módulo del vector entre la cara superior e inferior, es decir, el espesor mínimo de la probeta para cada instante del ensayo de SPT. La Fig. 8 muestra el espesor mínimo obtenido de esta forma, en función del desplazamiento de la bola. En esta figura se puede ver una etapa inicial, corta y abrupta, asociada a la reducción de espesor en la parte central de la probeta, atribuida a la penetración de la bola en la misma. En efecto, se aprecia que la etapa inicial

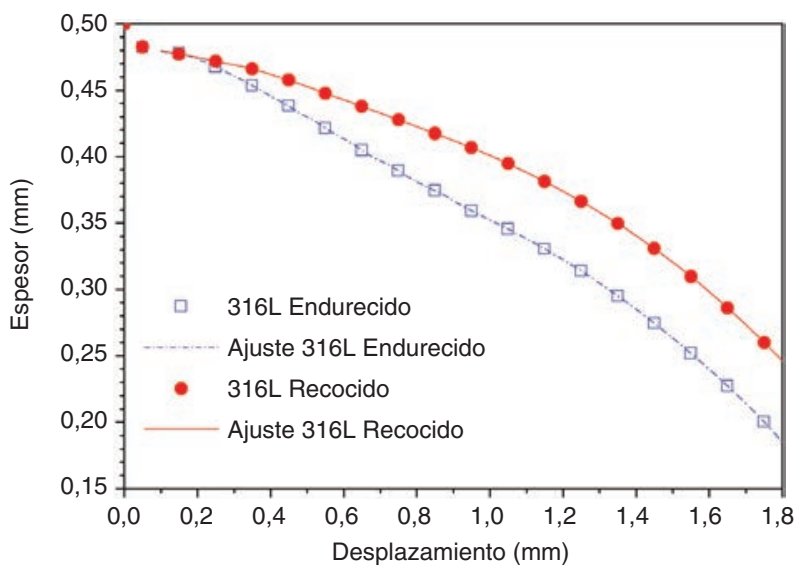

Figura 8. Espesor mínimo de la probeta en función del desplazamiento de la bola según MEF.

tanto de la Fig. 6 (zona central) como de la Fig. 8 (toda la probeta) coinciden, corroborando que la máxima reducción de espesor en los primeros instantes del ensayo se registra en la parte central de la probeta. Seguidamente, el espesor mínimo se desplaza a otra zona según se impone el desplazamiento de la bola. Este espesor mínimo tiende a desplazarse hacia el exterior de la probeta, dando lugar a una variación no lineal del área de trabajo. Es decir, el espesor mínimo disminuye a medida que aumenta el desplazamiento de la bola; sin embargo, el área efectiva de deformación aumenta por el hecho de que el área que sustenta la carga es proporcional a una corona de radio interior variable, dependiente del radio de la bola y su posición en cada instante. Ahora bien, aunque inicialmente la penetración de la bola viene a coincidir en ambos materiales, sus diferentes comportamientos de deformación plástica llevan a separar sus curvas de espesor mínimo. Así, se observa que el material que posee un gran endurecimiento por deformación, el recocido, posee una variación del espesor más homogénea que el endurecido, que es más localizada.

Después de deformar ligeramente la zona central de la probeta y en la zona de apoyos, la deformación se concentra en la zona de rotura, cercana a la zona donde el disco deja de tocar la bola. Desde la zona exterior hasta la interior y cogiendo paulatinamente más material, cada aleación llega a deformar mayor o menor zona dependiendo de su capacidad de endurecimiento por deformación. Así, inicialmente y hasta unas 250 micras de penetración de la bola no se observa diferencia en la deformación de uno $\mathrm{u}$ otro acero. Sin embargo, a partir de este valor, el acero recocido sigue incorporando más material a su deformación global, disminuyendo la velocidad de reducción de espesor localizada en la zona de rotura. Es decir, el volumen deformado del acero $316 \mathrm{~L}$ recocido es mayor, a igualdad de penetración de la bola, y por ello el espesor en la zona de rotura es mayor 
que el del acero $316 \mathrm{~L}$ endurecido. Una vez que el endurecimiento por deformación ya no puede incorporar más volumen de material deformado, la curva sigue una reducción de espesor paralela a la del material endurecido. En el caso que nos ocupa del acero $316 \mathrm{~L}$, la variación de espesor se ve afectada hasta aproximadamente $1,1 \mathrm{~mm}$. A partir de aquí las curvas de espesor mínimo de los dos materiales extremos aceleran su caída paralelamente indicando posible daño y comienzo de la fractura del material.

Este comportamiento de deformación contrasta con el de ensayos de tracción convencionales en los que la reducción de espesor es uniforme en toda la probeta desde el YS hasta el UTS, y el volumen de trabajo está perfectamente definido por la probeta de tracción (área x longitud). En cambio, en una probeta de SPT la reducción de espesor uniforme sólo se daría en cierto intervalo de desplazamiento de la bola. Este intervalo, como muestra la comparación de sus curvas, es función del endurecimiento por deformación del material. De hecho, dada una posición de la bola, dos materiales se encontrarán con distinto espesor mínimo, que es el que soporta toda la carga. Aun soportando ambos materiales la misma tensión, como la carga medida en el ensayo SPT depende de su espesor, ésta es mayor en el material que más endurece. Es esta una de las ideas que lleva a una necesidad imperativa de conocer la evolución del espesor si se quieren obtener un límite elástico y una resistencia máxima de este ensayo. Además, se ve alterada también la velocidad de deformación local, en la zona de rotura, que se asocia a la velocidad de deformación verdadera real o efectiva para el ensayo.

La Fig. 8 muestra también las líneas de ajuste a un polinomio de grado 4 de las dos curvas de espesor de ambos materiales en función del desplazamiento de la bola. A falta de obtener una relación funcional con significado físico y carácter predictivo en función de los parámetros mecánicos del material y geométricos del ensayo SPT, estos polinomios servirán para acotar el comportamiento del espesor de cualquier probeta de acero $316 \mathrm{~L}$. Las relaciones entre el espesor, $e$, y el desplazamiento, $d$, se dan a continuación:

$$
\begin{aligned}
\text { e }(316 \mathrm{~L} \text { Endurecido }) & =-0,049 \mathrm{~d}^{4}+0,1536 \mathrm{~d}^{3}-0,1737 \mathrm{~d}^{2} \\
& -0,0714 \mathrm{~d}+0,4925 \\
\text { e (316L Recocido }) & =-0,023 \mathrm{~d}^{4}+0,0605 \mathrm{~d}^{3}-0,0916 \mathrm{~d}^{2} \\
& -0,0296 \mathrm{~d}+0,484
\end{aligned}
$$

donde $d$ toma valores entre 0,17 y $1,8 \mathrm{~mm}$. Estos ajustes permiten mostrar cómo para una penetración de $1 \mathrm{~mm}, e$ (316L Endurecido) $=0,352 \mathrm{~mm}$ y $e(316 \mathrm{~L}$ Recocido $)=0,400 \mathrm{~mm}$. Es decir, el espesor es un 13,7\% mayor en el material recocido que en el endurecido a este desplazamiento. Esta diferencia de espesores aumenta más todavía a mayores desplazamientos de la bola.

Dado que se ha descrito el comportamiento de variación de espesor de dos casos extremos de acero $316 \mathrm{~L}$, recocido y endurecido, es de esperar que cualquier otro tratamiento termomecánico que produjera resistencias intermedias se describiera mediante otra curva situada entre las dos descritas por Eq. (1) y Eq. (2). Se espera ir corroborando esta conclusión con futuros tratamientos y ensayos SPT realizados sobre diferentes $316 \mathrm{~L}$ fabricados por diversas técnicas y sometidos a diferentes procesados.

\section{CONCLUSIONES}

Se ha determinado la variación del espesor de probetas de acero 316L en el ensayo de Small Punch Test (SPT), en dos tratamientos muy distintos, extremos (recocido y endurecido), mediante el empleo de un modelo de elementos finitos, MEF. De este trabajo se han obtenido las siguientes conclusiones:

- Se ha validado un modelo numérico de elementos finitos para el ensayo SPT, con especial énfasis en el cálculo del espesor de las probetas comparando con ensayos experimentales de acero 316L obtenido por fabricación aditiva por láser SLM.

- Las macrografías del acero 316L SLM después de ensayar muestran que la deformación ha sido muy heterogénea destacando 3 zonas. La zona cercana a los apoyos (zona amplia, corona entre radios 1,2 y $2 \mathrm{~mm}$ ) apenas contribuye a la deformación de la probeta. Otra zona es la central, o copete (entre radios 0 y 0,4 ), muy influida por el rozamiento, disminuyendo la alimentación de material a la zona de rotura, entre estas dos zonas. Finalmente, la zona de rotura, la cual depende del endurecimiento por deformación del material.

- El modelo de elementos finitos se ha alimentado con dos casos bien diferenciados de acero $316 \mathrm{~L}$, uno recocido, blando y con gran endurecimiento por deformación, y otro previamente endurecido por deformación, resistente y con poco endurecimiento por deformación residual. El modelo predice las variaciones no lineales de espesor según procede el ensayo tanto de la zona central como de la zona de rotura y para cada material.

- La diferencia de espesores en la zona de rotura entre ambos materiales es significativa (un $13,7 \%$ a $1 \mathrm{~mm}$ de desplazamiento). El desconocimiento de este comportamiento del espesor para cada material dificulta la predicción de datos mecánicos relativos a ensayos de tracción.

- Las diferencias en la variación del espesor para ambos materiales están directamente asociados a sus propiedades mecánicas y, especialmente, 
el endurecimiento por deformación. Materiales dúctiles con gran endurecimiento por deformación permiten obtener zonas deformadas más amplias donde el espesor varía de forma más homogénea.

- La variación de espesores a lo largo del ensayo SPT sigue una línea que se separa en el acero 316L Recocido, desde 0,25 mm, gracias a su endurecimiento por deformación. Otro acero 316L en condiciones distintas de tratamiento térmico mostraría un comportamiento entre estos dos extremos.

\section{AGRADECIMIENTOS}

Este trabajo contó con el apoyo del Ministerio de Economía, Industria y Competitividad de España bajo el proyecto MAT2015-68919-C3-1-R (MINECO/FEDER). Marta Álvarez-Leal agradece al MINECO un contrato FPI BES-2013-063963, D. David Sánchez-Ávila agradece a la Universidad Nebrija por el apoyo de la Beca Predoctoral y su convenio de colaboración con CENIM, CSIC.

\section{REFERENCIAS}

Alcelay, J.I., Peña, E., Al Omar, A. (2016). Estudio del comportamiento termo-mecánico de un acero microlaeado de medio carbono durante un proceso de conformado en caliente usando una red neuronal artificial. Rev. Metal. 52 (2), e066. https://doi.org/10.3989/revmetalm.066.

Altstadt, E., Ge, H.E., Kuksenko, V., Serrano, M., Houska, M., Lasan, M., Bruchhausen, M., Lapetite, J.-M., Dai, Y. (2016a). Critical evaluation of the small punch test as a screening procedure for mechanical properties. J. Nucl. Mater. 472, 186-195. https://doi.org/10.1016/j. jnucmat.2015.07.029.

Altstadt, E., Serrano, M., Houska, M., García-Junceda, A. (2016b). Effect of anisotropic microstructure of a $12 \mathrm{Cr}$-ODS steel on the fracture behaviour in the small punch test. Mat. Sci. Eng. A-Struct. 654, 309-316. https:// doi.org/10.1016/j.msea.2015.12.055

Arroyo, B., Álvarez, J.A., Lacalle, R., Uribe, C., García, T.E., Rodríguez, C. (2017). Analysis of Key Factors of Hydrogen Environmental Assisted Cracking Evaluation by Small Punch Test on Medium and High Strength Steels. Mat. Sci. Eng. A-Struct. https://doi.org/10.1016/j.msea.2017.03.006.

Autillo, J., Contreras, M.A. Betegón, C., Rodríguez, C., Belzunce, F. (2006). Utilización del Ensayo Miniatura de Punzonamiento (Small Punch test) en la Caracterización Mecánica de Aceros. Anales de La Mecánica de La Fractura $1,77-83$.

CEN Workshop Agreement (2006). Small Punch Test Method for Metallic Materials. CEN, Belgium, Brussels.

Cisneros-Belmonte, M., Ruiz-Román, J.M., García-Cambronero, L.E. (2016). Aceros sinterizados al Mo con gradiente funcional; Functionally Graded Mo sintered steels. Rev. Metal. 52 (4). https://doi.org/10.3989/revmetalm.081.

Díaz, L.A., López, M.E., Rodríguez, C., Belzunce, J., Torrecillas, R. (2009). Síntesis coloidal de materiales nanoestructurados de Al-ZrAl3: Propiedades mecánicas mediante el ensayo miniatura de punzonado. Rev. Metal. 45 (4), 256 266. https://doi.org/10.3989/revmetalm.0813.

Du, P., Ling, X., Zhou, Z., Xu, T. (2010) - Study on Influence Factors of Small Punch Test to Estimate the Yield Strenght by Energy model, $1^{\text {st }}$ Internacional Conference SSTT, Ostrava, República Checa.
Fleury, E., Ha, J.S. (1998). Small punch tests to estimate the mechanical properties of steels for steam power plant: I. Mechanical strength. Int. J. Pres. Ves. Pip. 75 (9), 699-706. https://doi.org/10.1016/S0308-0161(98)00074-X.

García-Infanta, J.M., Zhilyaev, A.P., Sharafutdinov, A., Ruano, O.A., Carreño, F. (2009). An evidence of high strain rate superplasticity at intermediate homologous temperatures in an $\mathrm{Al}-\mathrm{Zn}-\mathrm{Mg}-\mathrm{Cu}$ alloy processed by highpressure torsion. J. Alloy Compd. 473 (1-2), 163-166. https://doi.org/10.1016/j.jallcom.2008.06.006.

García, T.E., Rodríguez, C., Belzunce, F.J., Suárez, C. (2014). Estimation of the mechanical properties of metallic materials by means of the small punch test. J. Alloy Compd. 582, 708-717. https://doi.org/10.1016/j.jallcom.2013.08.009.

Gu, C., Bao, Y., Lin, L. (2017). Cleanliness distribution of highcarbon chromium bearing steel billets and growth behavior of inclusions during solidification. Rev. Metal. 53 (1), e089. https://doi.org/10.3989/revmetalm.089.

Haroush, S., Priel, E., Moreno, D., Busiba, A., Silverman, I., Turgeman, A., Shneck, R., Gelbstein, Y. (2015). Evaluation of the mechanical properties of SS-316L thin foils by small punch testing and finite element analysis. Mater. Design 83, 75-84. https://doi.org/10.1016/j.matdes.2015.05.049.

Lucas, G.E., Okada, A., Kiritani, M. (1986). Parametric analysis of the disc bend test. J. Nucl. Mater. 141-143 (Part 1), 532-535. https://doi.org/10.1016/S0022-3115(86)80096-4.

Ma, Y.W., Yoon, K.B. (2010). Assessment of tensile strength using small punch test for transversely isotropic aluminum 2024 alloy produced by equal channel angular pressing. Mat. Sci. Eng. A-Struct. 527 (16-17), 3630-3638. https:// doi.org/10.1016/j.msea.2010.02.057.

Manahan, M.P., Argon, A.S., Harling, O.K. (1981). The development of a miniaturized disk bend test for the determination of postirradiation mechanical properties. J. Nucl. Mater. 104, 1545-1550. https://doi. org/10.1016/0022-3115(82)90820-0.

Mao, X., Takahashi, H. (1987). Development of a furtherminiaturized specimen of $3 \mathrm{~mm}$ diameter for tem disk ( $3 \mathrm{~mm}$ ) small punch tests. J. Nucl. Mater. 150 (1), 42-52. https://doi.org/10.1016/0022-3115(87)90092-4.

Martínez-Pañeda, E., Cuesta, I.I., Peñuelas, I., Díaz, A., Alegre, J.M. (2016). Damage modeling in Small Punch Test specimens. Theor. Appl. Fract. Mec. 86 (Part A), 51-60. https://doi.org/10.1016/j.tafmec.2016.09.002.

Medina, S.F., Quispe, A., Gómez, M. (2015). Precipitation model in microalloyed steels both isothermal and continuous cooling conditions. Rev. Metal. 51 (4), e056. https:// doi.org/10.3989/revmetalm.056.

Misawa, T., Adachi, T., Saito, M., Hamaguchi, Y. (1987). Small punch tests for evaluating ductile-brittle of irradiated ferritic steels. J. Nucl. Mater. 150 (2), 194-202. https://doi. org/10.1016/0022-3115(87)90075-4

Moreno, M.F., Bertolino, G., Yawny, A. (2016). The significance of specimen displacement definition on the mechanical properties derived from Small Punch Test. Mater. Design 95, 623-631. https://doi.org/10.1016/j.matdes.2016.01.148.

Pascual, M., Salas, F Carcel, FJ., Perales, M., Sánchez, A. (2010). TIG AISI-316 welds using an inert gas welding chamber and different filler metals: Changes in mechanical properties and microstructure. Rev. Metal. 46 (6), 493-498. https://doi.org/10.3989/revmetalmadrid.1005.

Puchi-Cabrera, E.S., Saya-Gamboa, R.A., La Barbera-Sosa, J.G., Staia, M.H., Ignoto-Cardinale, V., Berríos-Ortíz, J.A., Mesmacque, G. (2007). Vida a la fatiga de juntas soldadas del acero inoxidable AISI 316L obtenidas mediante el proceso GMAW*. Rev. Metal. 43 (3), 215-227. https://doi. org/10.3989/revmetalm.2007.v43.i3.67.

Rasche, S., Strobl, S., Kuna, M., Bermejo, R., Lube, T. (2014). Determination of Strength and Fracture Toughness of Small Ceramic Discs Using the Small Punch Test and the Ball-on-three-balls Test. Procedia Mater. Sci. 3, 961-966. https://doi.org/10.1016/j.mspro.2014.06.156.

Rodríguez, C.C., Belzunce, F.J. (2012). Estudio de la idoneidad del ensayo miniatura de punzonado para la caracterizacion mecanica de los aceros y de sus uniones soldadas. Dyna $79,53-60$ 
Rodríguez, C., Cuesta, I.I., Maspoch, M.L., Belzunce, F.J. 2016). Application of the miniature small punch test for the mechanical characterization of polymer materials. Theor. Appl. Fract. Mec. 86, 78-83. https://doi. org/10.1016/j.tafmec.2016.10.001

Saucedo-Muñoz, M.L., Komazaki, S., Hashida, T., LopezHirata, V.M. (2015). Small Punch Creep test in a 316 austenitic stainless steel. Rev. Metal. 51 (1), e034. https://doi. org/10.3989/revmetalm.034

Sevim, I. (2016). An experimental study on fracture toughness of resistance spot welded galvanized and ungalvanized DP 450 steel sheets used in automotive body. Rev. Metal. 52 (3), e072. https://doi.org/10.3989/revmetalm.072.

Simonovski, I., Holmström, S., Bruchhausen, M. (2017). Small punch tensile testing of curved specimens: Finite element analysis and experiment. Int. J. Mech. Sci. 120, 204-213. https://doi.org/10.1016/j.ijmecsci.2016.11.029.

Štrbac, N., Markovi, I., Mitovski, A., Balanovi, L. (2017). The possibilities for reuse of steel scrap in order to obtain blades for knives. Rev. Metal. 53 (1), e086. https://doi. org/10.3989/revmetalm.086.

Tsisar, V., Schroer, C., Wedemeyer, O., Skrypnik, A., Konys, J. (2014). Corrosion behavior of austenitic steels 1.4970 , $316 \mathrm{~L}$ and 1.4571 in flowing LBE at 450 and $550^{\circ} \mathrm{C}$ with
10-7 mass\% dissolved oxygen. J. Nucl. Mater. 454 (1-3), 332-342. https://doi.org/10.1016/j.jnucmat.2014.08.024.

Turba, K., Hurst, R., Hähner, P. (2013). Evaluation of the ductile-brittle transition temperature in the NESC-I material using small punch testing. Int. J. Pres. Ves. Pip. 111-112, 155-161. https://doi.org/10.1016/j.ijpvp.2013.07.001.

Yang, S., Ling, X., Zheng, Y. (2017). Creep behaviors evaluation of Incoloy $800 \mathrm{H}$ by small punch creep test. Mat. Sci. Eng. A-Struct. 685, 1-6. https://doi.org/10.1016/j. msea.2016.12.092.

Zhao, G., Huang, Q., Zhou, C., Zhang, Z., Ma, L., Wang, X. (2016). Experiment and simulation analysis of roll-bonded Q235 steel plate. Rev. Metal. 52 (2), e069. https://doi. org/10.3989/revmetalm.069.

Zhong, Y., Rännar, L.-E., Liu, L., Koptyug, A., Wikman, S., Olsen, J., Cui, D., Shen, Z. (2017). Additive manufacturing of $316 \mathrm{~L}$ stainless steel by electron beam melting for nuclear fusion applications. J. Nucl. Mater. 486, 234-245. https:// doi.org/10.1016/j.jnucmat.2016.12.042.

Zhou, X., Pan, W., Mackenzie, D. (2013). Material plastic properties characterization by coupling experimental and numerical analysis of small punch beam tests. Comput. Struct. 118, 59-65. https://doi.org/10.1016/j. compstruc.2012.07.002. 